\title{
Cross correlation of UHECRs and local matter distribution taking into account the energy attenuation due to interaction with the cosmic background radiation
}

\author{
C. S. L. Pinto ${ }^{a}$, V. Braga $^{b}$, R. M. de Almeida ${ }^{a *}$, J. R. T. de Mello Neto ${ }^{b}$ \\ ${ }^{a}$ EEIMVR - Universidade Federal Fluminense, RJ, Brazil \\ ${ }^{b}$ Instituto de Física, Universidade Federal do Rio de Janeiro, RJ, Brazil \\ E-mail: rmenezesdid.uff.br
}

\begin{abstract}
The arrival direction distribution of ultra-high energy cosmic rays (UHECRs) is a key element to understand their sources and composition as well the galactic and extragalactic magnetic fields. The particles that arrive on Earth with energies above $\sim 50 \mathrm{EeV}$ must be originated in sources nearby us as a result of the GZK effect. Furthermore, if these particle are protons and the magnetic fields along their propagations until Earth are not too large, we expect that their arrival directions are correlated with their sources. The cross-correlation used in cosmology is a good tool for studying such anisotropies in the cosmic ray data. In this work, assuming a proton composition, we modify the Landy-Szalay cross-correlation estimator in order to take into account the energy losses in the cosmic ray propagation due to its interaction with the background radiation. Using Monte Carlo simulations, we have compared its efficiency with relation to the original estimator, testing the correlation of the simulated events arrival directions with sources from the 2MRS catalog. We show that even in case of absence of significant signal, the modified estimator has the capability to constrain the proton fraction of the experimental data provided that the detection efficiency of the astrophysical scenario under consideration is maximal.
\end{abstract}

35th International Cosmic Ray Conference - ICRC2017

10-20 July, 2017

Bexco, Busan, Korea

${ }^{*}$ Speaker. 


\section{Introduction}

The arrival direction distribution of ultra-high energy cosmic rays (UHECRs, particles with energy exceeding $E>10^{19} \mathrm{eV}$ ) is an important observable to understand the origin and composition of such high energy particles as well as their propagations through the inter-estelar media.

As cosmic rays propagate, the highest-energy particles interact strongly with the ubiquitous cosmic background radiation and lose energy by photo-pion production, in case of proton, or photodisintegration in case of heavier nuclei. This mechanism, known as GZK effect, was predicted by Greisen [1], Zatsepin and Kuz'min [2] more than 50 years ago and would produce a suppression of the particle flux above $\sim 50 \mathrm{EeV}$, resulting in a steepening of the energy spectrum. The Pierre Auger Collaboration reported a confirmation of this flux suppression beyond any doubt [?, 4] although its origin could also be due the maximum injection energy of the particles in the sources. If the GZK mechanism is responsible for this flux suppression, the highest energy particle should be produced in our local universe. Further, if they are protons and the galactic and extra-galactic magnetic fields are not to extreme, we expect that their arrival directions should be correlated with the clustered local matter distribution. Several searches for anisotropy aiming to correlate the arrival directions of UHECRs with astrophysical objects have been performed in the past $[5,6]$ and in recent years $[9,10,11,12,13,14,15,16]$ with different UHECR samples, statistical methods and source populations.

Let $E_{f}$ be the degraded proton energy at Earth after the propagation from the source with initial energy $E_{i}$. Assuming a power-law injection spectrum, the attenuation factor $\omega_{G Z K}\left(z, E_{t h}\right)$ is defined as the fraction of particles which originally had an energy above a given threshold $E_{t h}$ that still have an energy above that value after traveling a distance $z$. A direct way to compute this factor is to follow the trajectories of many individual protons with initial energies distributed according a power law spectrum. Figure 1 shows the attenuation factor $\omega_{G Z K}\left(z, E_{t h}\right)$ obtained with CRPropa 3 [17] for protons with different energy thresholds and injection power law spectrum $d N / d E \propto E^{-2.2}$ with a maximum injection energy of $10^{21} \mathrm{eV}$.

By assuming equal intrinsic luminosity and homogenous source distribution, one finds that the fraction $\Omega_{G Z K}$ of the events observed above a given energy threshold which were originated in sources farther than a distance $z$ is

$$
\Omega_{G Z K}\left(z ; E_{f}\right)=\frac{\int_{z}^{\infty} \omega_{G Z K}\left(z ; E_{f}\right) d z^{\prime}}{\int_{0}^{\infty} \omega_{G Z K}\left(z ; E_{f}\right) d z^{\prime}} .
$$

For a given energy threshold, the GZK horizon $R_{G Z K}$ is defined as the distance such that 1 $\Omega_{G Z K}\left(R, E_{f}\right)=0.9$, being $R_{G Z K} \sim 130 \mathrm{Mpc}$ for an energy threshold $E_{t h}=70 \mathrm{EeV}$ and $R_{G Z K} \sim 200$ Mpc for $E_{t h}=60 \mathrm{EeV}$. Therefore, the highest energy cosmic ray source distribution is generally assumed to be the local matter distribution. The local universe is well represented by measurements performed by the 2MASS collaboration which imaged the whole sky in the near-infrared $(\mathrm{J}, \mathrm{H}$, and K bands) [18].

A subset of the 2MASS catalog with the brightest objects $\left(M_{k}<-24.13\right)$ called 2MRS, restricted to $|b|<10^{\circ}$ to avoid catalog incompleteness from Galactic extinction [19] is an example of a good catalog for cross-correlation studies and is used along this work. It is a flux-limited catalog, that is, it contains objects up to certain apparent luminosity in the corresponding band. For this 


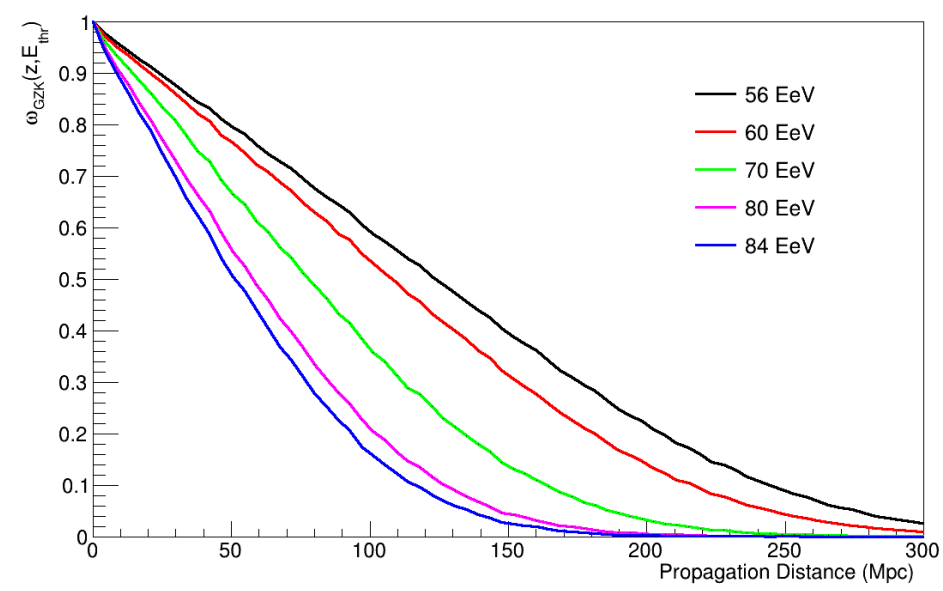

Figure 1: Attenuation factor $\omega_{G Z K}\left(z, E_{t h}\right)$ obtained with CRPropa 3 for protons with different energy thresholds as a function of the propagated distance.

reason, it represents incorrectly the space distribution of sources, with much more sources at close distances than at large ones, because only bright sources are seen at large distances. In this work we restrict the analysis to sources with absolute magnitudes $M<-25.0$. The resulting sample is complete in distance for test the cross-correlation with events with energy above $70 \mathrm{EeV}$ since the $R_{G Z K} \sim 130 \mathrm{Mpc}$ is relatively small. On the other hand, for particles with $E>60 \mathrm{EeV}$, the GZK horizon $R_{G Z K} \sim 200 \mathrm{Mpc}$ and the distribution of the number of sources for the samples selected with both absolute magnitudes do not increase $\sim D^{2}$, as it would be expected in case of homogeneous source distribution. Therefore, we complete these samples by including random isotropic sources such as the resulting distributions of the number of sources $(n)$ are $d n / d D \sim D^{2}$.

A non- zero cross-correlation between UHECRs and a given catalog proves the anisotropy of UHECRs with no assumption on the relation between UHECR sources and the catalogue. LandySzalay have shown in [20] that their cross-correlation estimator has a smaller variance, optimizing the efficiency to detect possible anisotropies. However, assuming that the highest energy cosmic rays are dominated by protons originated in sources nearby us and that the magnetic fields are not too large, we claim that a modification in the Landy-Szalay cross-correlation estimator, in order to taking into account informations about the source distances, could by principle enhance its anisotropy detection efficiency.

Therefore, we modify the Landy-Szalay cross correlation estimator in order to take into account the proton energy losses along its propagation due to interactions with the cosmic background radiation. Section 2 presents a brief description of the Landy-Szalay estimator as well as a detailed discussion of our modification while section 3 shows a comparison of the anisotropy detection efficiency between the original and the modified estimator. The conclusions of this work are presented in the end of this paper. 


\section{Cross correlation analyses methods}

\subsection{Landy-Szalay estimator}

The cross-correlation method is a technique designed to measure the clustering level of a certain set of directional data through correlation with another set of directions. This technique originally appeared in the context of cosmology aiming to quantify the clustering level of objects in the universe and today is a very useful tool in the study of anisotropy of ultra high energy cosmic rays.

There are several estimators to accomplish this task. The Landy-Szalay cross-correlation estimator between catalogues 1 and 2 is defined as

$$
\omega(\theta)=\frac{N_{D_{1} D_{2}}-N_{D_{1} R_{2}}-N_{R_{1} D_{2}}+N_{R_{1} R_{2}}}{N_{R_{1} R_{2}}},
$$

where $N_{D_{1} D_{2}}$ is the number of pairs between the two data samples as a function of $\theta, N_{D_{1} R_{2}}$ and $N_{D_{2} R_{1}}$ are the number of pairs in the given bin of $\theta$ between each data sample and the random sample with the exposure of the other data sample, and $N_{R_{1} R_{2}}$ is the number of random-random pairs with exposures of catalogs 1 and 2 , as a function of $\theta$. In this work, we consider that $D_{1}$ is composed by events simulated with the Pierre Auger Collaboration exposure, $D_{2}$ is a sample of sources from the $2 \mathrm{MRS}$ catalogue while the random sets $R_{1,2}$ contain the exposure information for the two catalogs. Notice that this estimator vanishes if $D_{1}$ or $D_{2}$ is isotropic, because in these cases $D_{1}=R_{1}$ or $D_{2}=R_{2}$.

A possible choice of a global estimator for the cross-correlation between samples $D_{1}$ and $D_{2}$ that takes into account the statistical penalty for searches in different angular scales is

$$
\chi^{2}=\frac{1}{N_{\text {bins }}} \sum_{i=1}^{N_{\text {bins }}}\left(\frac{\omega(\theta)-<\omega(\theta)>}{\sigma_{\text {iso }}}\right)^{2},
$$

where $\omega(\theta),<\omega(\theta)_{i s o}>$ and $\sigma_{i s o}$ are, respectively, the value of the estimator from the observed data, the average and standard deviation from the isotropic $\omega(\theta)$ expectations, all of them evaluated for a given angular scale $\theta$. In practice we obtain the $\left\langle\omega(\theta)_{\text {iso }}>\right.$ and $\sigma_{\text {iso }}$ values using simulated isotropic skies with the same number of events and exposure of the data. $N_{\text {bins }}$ is the number of bins for searches in different angular scales $\theta$.

\subsection{Modified Landy-Szalay estimator}

As mentioned before, in the context of ultra high energy cosmic rays, particles are subject to the GZK effect, which reduces the volume of the universe for the origin of such particles. Since the original Landy-Szalay estimator does not take it into account we propose here a modification in this estimator aiming to increase its detection efficiency, although we have to pay the price of assuming a primary composition. It consists in replacing the counting of the number of pairs between samples $X_{1}$ and $D_{2}$ by including a weight that depends on the distances of the sources from the catalogue $D_{2}$ :

$$
N_{X_{1} D_{2}}(\theta)=\sum_{i} \sum_{j} p_{j}\left(D_{j}\right) \times \Theta\left(\theta_{i j}\right)
$$


where the index $i$ runs over all elements of the sample $X_{1}\left(D_{1}\right.$ or $R_{1}$ in equation 2.1), $j$ runs over all elements of the catalog $D_{2}, \Theta\left(\theta_{i j}\right)$ is the heaviside step function and $\theta_{i j}$ is the angle between a pair of directions from $X_{1}$ and $D_{2}$. The weight $p_{j}$ depends of the attenuation factor $\omega_{G Z K}\left(z, E_{t h}\right)$ and, therefore, is proportional to the probability of a cosmic ray to reach the Earth with energy $E>E_{t h}$,

$$
p_{i}(D)=\frac{\omega_{G Z K\left(D, E_{t h}\right)}}{\omega_{G Z K\left(D_{\max }, E_{t h}\right)}} \frac{D_{\max }^{2}}{D^{2}},
$$

where $D$ is the distance from the astrophysical object and $D_{\max }$ is the maximum distance of an object in the catalog. Notice that even with this modification, this estimator also vanishes when the sample $D_{1}$ is isotropic. The test of isotropy of the $D_{1}$ sample, taking into account the searches in different angular scales, is performed using the same global estimator $\chi^{2}$ used for the original Landy-Szalay estimator, defined by Eq. (2.2).

\section{Simulations and results}

It is expected that a realistic set of cosmic rays contains a mixture of isotropic and anisotropic events, that is, signal and noise. Also, the number of events contained in each set is equally important for the analysis of anisotropies, due to the low flux of cosmic rays on the extreme-energy range. The impact of the combined effect of the dilution of the signal in the background and the sample size on the power of the test was evaluated for several sets of simulated events with energy thresholds of $60 \mathrm{EeV}$ and $70 \mathrm{EeV}$ generated from sources of the 2MRS catalog with absolute magnitudes $M<-25.0$. Due to the deflections in the galactic and extragalactic magnetic fields, we assume that only protons will be correlated with their sources. Therefore, the isotropic background is formed by the contribution of heavier nuclei to the cosmic ray flux.

The algorithm used to evaluate the detection efficiency is described in the following. First, the 2MRS catalogue is used to define possible sources of UHECRs. Among the sources, one is randomly chosen and a proton is accepted/rejected at Earth according to the attenuation factor $\omega_{G Z K}\left(D, E>E_{t h}\right)$ and the exposure of the experiment. As mentioned before, we used in this work the exposure of the Pierre Auger Observatory, given by [22].

The effects of the magnetic fields along the cosmic ray propagation are included by performing a gaussian smearing in the cosmic ray arrival direction with an angular scale $\sigma=5^{\circ}$. This propagation process is repeated until we obtain the desired proton fraction $f_{p}$ of the simulated $N$ events. The remaining $1-f_{p}$ events arrival directions are isotropically distributed in the sky according to the Pierre Auger Collaboration exposure. We performed this algorithm for different proton fractions $\left(f_{p}=20 \%, 40 \%, 60 \%, 80 \%\right.$ and $\left.100 \%\right)$ and total number of events $(N=20,40,60,80$ and 100).

Figure 2 presents a comparison between the detection efficiency of the test as a function of the proton fraction $f_{p}$ for the original Landy-Szalay estimator (dashed lines) and our modified version (solid lines) considering a energy threshold of $E>60 \mathrm{EeV}$. The detection efficiency is defined as $P=\frac{N\left(\chi^{2}>\chi_{\text {iso, }, 99 \%}^{2}\right)}{N_{\text {total }}}$, where $N_{\text {total }}$ is the number of simulated skies for a given scenario (total number of events and fraction of protons in the data set), i.e., the detection efficiency is the fraction of simulated skies for which the estimators $\chi^{2}$ is larger than $\chi_{\text {iso,99\% }}^{2}$. The different colors show how the detection efficiency is changed by the total number of events $N$. 


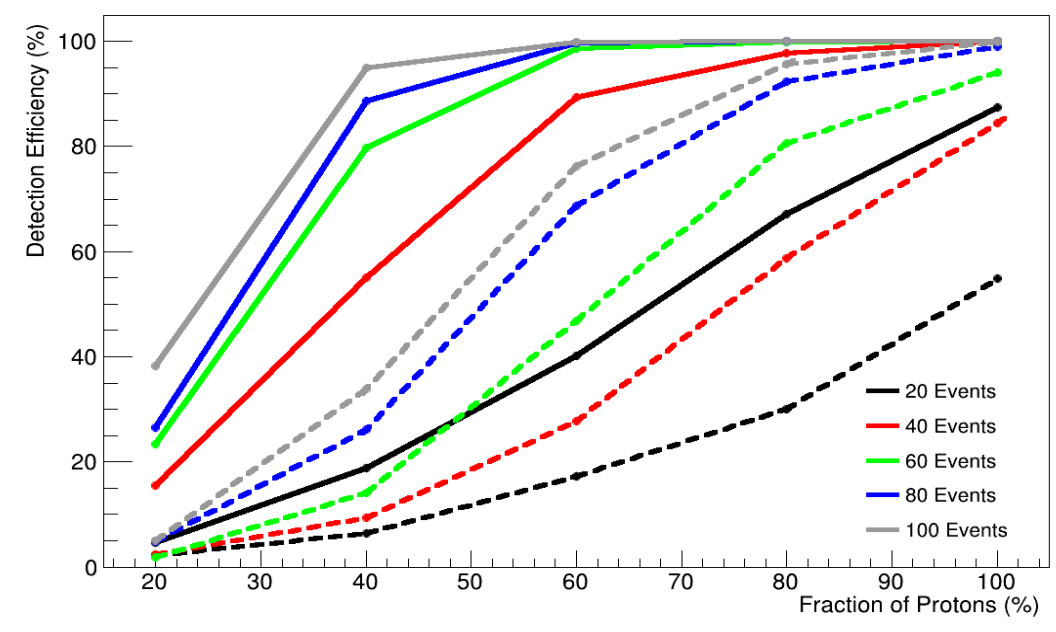

Figure 2: Comparison between the detection efficiency of the test as a function of the proton fraction $f_{p}$ for the original Landy-Szalay estimator (solid lines) and our modified version (dashed lines), using 2MRS sources selected with $M<-25.0$ and considering a energy threshold of $E>60 \mathrm{EeV}$. The different colors show how the detection efficiency is changed by the total number of events $N$.

Figure 3 presents the same for an energy threshold of $E>70 \mathrm{EeV}$. One can see that, as expected, the detection efficiency is largely enhanced when we introduce a weight that depends on the source distance in the Landy-Szalay estimator. However, it is important to notice that differently from the Landy-Szalay estimator, our modified one depends on the assumed primary composition which makes more difficulty this analysis since, for instance, the results obtained by the Pierre Auger Collaboration indicate a larger fraction of heavier nuclei as the energy increases. On the other hand, we emphasize that the weight factor given in equation 2.4 could also be modified to taking into account mixed primary composition and/or different source luminosities. This will be addressed in a different work. Furthermore, a negative cross-correlation result using a real data set composed by $N$ events, can be used to constrain the fraction of protons in the data for regions of this parameter (proton fraction) for which the detection efficiency equals $100 \%$.

\section{Conclusions}

In this work we have modified the Landy-Szalay cross-correlation estimator in order to taking into account the cosmic ray energy attenuation due to interactions with the cosmic background radiation. By assuming that the cosmic rays are protons originated in sources located in our local universe, described by $2 \mathrm{MRS}$ catalog, we compared the detection efficiencies of isotropy tests by using the cross-correlation Landy-Szalay estimator with and without this modification. As expected, there is a large enhacement on the power of the test with the introduction of this modification estimator, although we have to assume a given primary composition. Nevertheless, one should notice that the weight factor added to Landy-Szalay estimator can be suitably changed so as to take into account a mixed primary composition as well as scenarios in which sources have different intrinsic cosmic ray luminosities. Finally, we also have shown that even in case of absence 


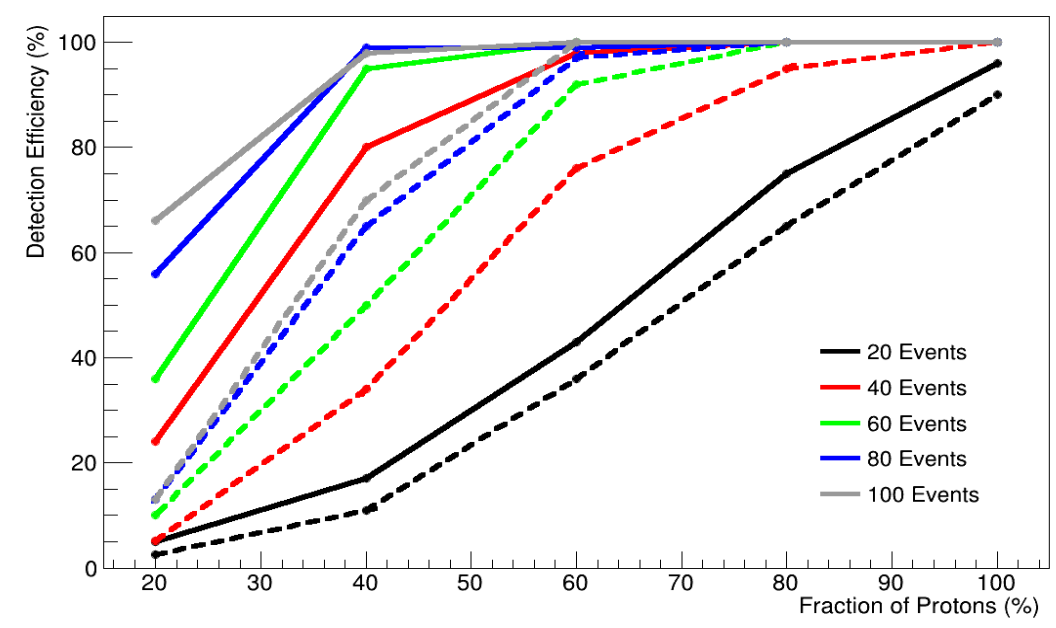

Figure 3: Comparison between the detection efficiency of the test as a function of the proton fraction $f_{p}$ for the original Landy-Szalay estimator (solid lines) and our modified version (dashed lines), using 2MRS sources selected with $M<-25.0$ and considering a energy threshold of $E>70 \mathrm{EeV}$. The different colors show how the detection efficiency is changed by the total number of events $N$.

of significant cross correlation signal, the modified Landy-Szalay estimator has the capability to constrain the proton fraction of a experimental data set provided that the detection efficiency of the astrophysical scenario under consideration is maximal.

\section{Acknowledgments}

This work was partially supported by the Conselho Nacional de Desenvolvimento Científico e Tecnológico - CNPq and by the Fundação Carlos Chargas Filho de Amparo à Pesquisa do Estado do Rio de Janeiro - FAPERJ.

\section{References}

[1] K. Greisen, End to the cosmic ray spectrum?, Phys. Rev. Lett. 16 (1966) 748.

[2] G. Zatsepin and V. Kuzmin, Upper limit of the spectrum of cosmic rays, JETP Lett. 4 (1966) 78.

[3] The Pierre Auger Collaboration, Physical Review Letters 101, 061101 (2008)

[4] Pierre Auger Collaboration, A. Schulz, "The measurement of the energy spectrum of cosmic rays above $3 \times 10^{17} \mathrm{eV}$ with the Pierre Auger Observatory", Proc. of 33rd Int. Cosmic Ray Conf., Rio de Janeiro, Brazil, 0769 (2013) , arXiv:1307.5059 [astro-ph.HE].

[5] P. Tinyakov and I. Tkachev, BL Lacertae are sources of the observed ultrahigh-energy cosmic rays, JETP Lett. 74 (2001) 445 [astro-ph/0102476].

[6] D. Gorbunov, P. Tinyakov, I. Tkachev and S.V. Troitsky, Evidence for a connection between gamma-ray and highest energy cosmic ray emissions by BL Lacs, Astrophys. J. 577 (2002) L93 [astro-ph/0204360]. 
[7] The Pierre Auger Collaboration, "Large scale distribution of ultra high energy cosmic rays detected at the Pierre Auger Observatory with zenith angles up to 80 degrees", ApJ 802, 111 (2015)

[8] The Pierre Auger Collaboration,"Searches for Anisotropies in the Arrival Directions of the Highest Energy Cosmic Rays Detected by the Pierre Auger Observatory", ApJ 804, 15 (2015)

[9] The Pierre Auger Collaboration, Update on the correlation of the highest energy cosmic rays with nearby extragalactic matter, Astroparticle Physics 34 (Dec., 2010) 314-326, [arXiv:1009.1855].

[10] T. Kashti and E. Waxman, Searching for a correlation between cosmic-ray sources above $1019 \mathrm{eV}$ and large scale structure, Journal of Cosmology and Astro-Particle Physics 5 (May, 2008) 006, [arXiv:0801.4516].

[11] A. A. Berlind, G. R. Farrar, and I. Zaw, Correlations Between Ultrahigh Energy Cosmic Rays and Infrared-luminous Galaxies, The Astrophysical Journal 716 (June, 2010) 914-917 [arXiv:0904.4276].

[12] H. Takami, T. Nishimichi, K. Yahata, and K. Sato, Cross-correlation between UHECR arrival distribution and large-scale structure, Journal of Cosmology and Astroparticle Physics 6 (June, 2009) 31, [arXiv:0812.0424].

[13] The Pierre Auger Collaboration, Astrophysical Sources of Cosmic Rays and Related Measurements with the Pierre Auger Observatory, ArXiv e-prints (June, 2009) [arXiv:0906.2347].

[14] The Pierre Auger Collaboration, Correlation of the Highest-Energy Cosmic Rays with Nearby Extragalactic Objects, Science 318 (Nov., 2007) 938, [arXiv:0711.2256].

[15] The Pierre Auger Collaboration, Correlation of the highest-energy cosmic rays with the positions of nearby active galactic nuclei, Astroparticle Physics 29 (Apr., 2008) 188-204, [arXiv:0712.2843].

[16] L. J. Watson, D. J. Mortlock, and A. H. Jaffe, A Bayesian analysis of the 27 highest energy cosmic rays detected by the Pierre Auger Observatory, Monthly Notices of the RAS 418 (Nov., 2011) 206-213, [arXiv:1010.0911].

[17] R. Alves Batista et al., CRPropa 3 - a Public Astrophysical Simulation Framework for Propagating Extraterrestrial Ultra High energy Particles, JCAP 05 (2016) 038, [arXiv:1603.07142]

[18] M. F. Skrutskie, R. M. Cutri, R. Stiening, M. D. Weinberg, S. Schneider, J. M. Carpenter, C. Beichman, R. Capps, T. Chester, J. Elias, J. Huchra, J. Liebert, C. Lonsdale, D. G. Monet, S. Price, P. Seitzer, T. Jarrett, J. D. Kirkpatrick, J. E. Gizis, E. Howard, T. Evans, J. Fowler, L. Fullmer, R. Hurt, R. Light, E. L. Kopan, K. A. Marsh, H. L. McCallon, R. Tam, S. Van Dyk, and S. Wheelock, The Two Micron All Sky Survey (2MASS), Astronom. J., 131:1163-1183, February 2006.

[19] Huchra J.P., Macri L.M., Masters K.L., Jarrett T.H., Berlind P., Calkins M., Crook A.C., Cutri R., Erdogdu P., Falco E., George T., Hutcheson C.M., Lahav O., Mader J., Mink J.D., Martimbeau N., Schneider S., Skrutskie M., Tokarz S., Westover M., The 2MASS Redshift Survey - Description and Data Release, Astrophys. J. Suppl. 199, 26 (2012)

[20] S.D. Landy and A. S. Szalay, ApJ. 412:64-71, 1993

[21] Glen Cowan, Statistical Data Analysis, Oxford University Press, 1998.

[22] P. Sommers, Cosmic ray anisotropy analysis with a full-sky observatory, Astroparticle Physics 14 (Jan., 2001) 271-286, [astro-ph/0004016]. 